\title{
STUDY ON PERFORMANCE OF ELECTROKINETIC FILTRATION USING ROTARY DRUM VACUUM FILTER
}

\author{
HIROSHI YUKAWA, KAZUMASA KOBAYASHI \\ AND MASARU HAKODA \\ Department of Chemical Engineering, Gunma University, \\ Kiryu 376
}

\begin{abstract}
It was found in our previous paper that an electrokinetic filtration could considerably increase the filtration flow rate in comparison with that of the conventional filtration, because electrophoresis in the slurry and electroosmosis in the cake were caused by the electric field. This method is especially applicable to the filtration of slurries such as bentonite slurry, which is difficult to filter by mechanical pressure because of gelatinous cake formation. In this experiment, an electrokinetic filter made by modifying an Oliver filter was used and its performance was investigated. Calcium carbonate, white clay and bentonite were used as suspended particles. As a result, it was confirmed that electrokinetic filtration is applicable to practical filtration and that the equation for the electrokinetic filtration proposed in this paper is applicable to the design of a practical filtration process.
\end{abstract}

\section{Introduction}

Recently, the application of interfacial electrokinetic phenomena to filtration has been studied by some investigators. Moulik et al..$^{3,4)}$ reported that the filtration rate was enhanced because of decreasing particle deposition on the filter medium in the case of clarifying filtration. A solid-liquid separation proess based on crossflow and electrofiltration was investigated by Henry et al. ${ }^{2)}$ to prevent deposition of particles on filter medium with a kaolin clay suspension and an oil-in-water emulsion. According to the previous investigation $^{\text {?) }}$ a batch electrokinetic filtration in which a D. C. electric field was applied to constantpressure filtration considerably enhanced the filtration flow rate in comparison with that of conventional filtration because electrophoresis occurring in the slurry decreased cake formation and electroosmosis in the filter cake increased the filtration flow rate at the same time. Therefore, this method is expected to be especially applicable to the practical filtration of slurries such as bentonite slurry, which is difficult to filter by mechanical pressure alone because of gelatinous cake formation. In this paper, the performance of electrokinetic filtration is examined in comparison with a conventional vacuum filter with calcium carbonate slurry, white clay slurry and bentonite slurry forming the gelatinous cake. The test filter used in this experi-

Received November 7, 1979. Correspondence concerning this article should be addressed to K. Kobayashi. ment was a rotary drum vacuum filter, modified by electrical attachments. As a result, it was confirmed that electrokinetic filtration is applicable to industrial operation. An equation for continuous electrokinetic filtration was derived based on the equation for batch electrokinetic filtration, and it was found to be applicable to practical filtration. The relation between filtrate volume and electric power consumption could be represented by an empirical equation similar to that of batch electrokinetic filtration ${ }^{7)}$.

\section{Equation for Continuous Electrokinetic Filtration}

The equation for batch electrokinetic filtration, which has been presented previously and confirmed experimentally ${ }^{7}$, is expressed as follows:

$$
d t / d V=\left(2 / K_{R}\right)\left(V+V_{0}\right)
$$

in which

$$
K_{R}=\frac{2\left(\Delta P_{H}+\Delta P_{E}\right) A^{2}}{\mu \alpha \nu}, \quad V_{0}=\frac{R_{m} A}{\alpha \nu}, \quad \nu=\nu_{0}\left(\frac{E_{c r}-E}{E_{c r}}\right)
$$

Equation (1) is rewritten in terms of filtrate volume per unit filtration area $v=V / A$ and fictitious filtrate volume per unit area $v_{0}=V_{0} / A$ as follows:

$$
d t / d v=2\left(v+v_{0}\right) / k_{R}
$$

in which

$$
k_{R}=\frac{K_{R}}{A^{2}}=\frac{2\left(\Delta P_{H}+\Delta P_{E}\right)}{\mu \alpha \nu}, \quad v_{0}=\frac{R_{m}}{\alpha \nu}
$$

In continuous filtration with a rotary drum filter, the 
filtration time $t$ at which the appointed unit filtration area rotates $i$ times is $i / N$ when $N$ is the number of revolutions per unit time. Therefore, the real filtration time $t_{r}=t F$ is $F(i / N)$ where $F$ is the submergence ratio of drum filter. It is assumed here that the cake formed on the rotary drum filter is scraped off every $i$-th revolution and that the filtration resistance of the filter cake on the filter surface does not change whether the filter surface is immersed or not. Integrating Eq. (3) under conditions that $v=0$ at $t=0$ and $v=v$ at $t=t_{r}$, the relationship between $v$ and $t$. in electrokinetic filtration for each $i$ revolutions is obtained under conditions of constant filtration pressure and constant strength of electric field as follows ${ }^{1,6)}$ :

$$
\frac{t_{r}}{v}=\frac{F(i / N)}{v}=\frac{v+2 v_{0}}{k_{R}}
$$

The equation for the filtration flow rate is given by Eqs. (3) and (5) as follows:

$$
\frac{d v}{d t}=\frac{k_{R}}{2 \sqrt{k_{R} F(i / N)+v_{0}{ }^{2}}}
$$

Although the filtrate flow through the cake formed on the surface of a rotary drum filter is not precisely onedimensional, it may be considered to be one-dimensional when the thickness of the filter cake is negligibly small compared with the diameter of the drum filter ${ }^{5)}$.

\section{Experimental Apparatus and Procedure}

The outline of the experimental apparatus and the detailed diagram of the rotary drum filter are shown in Figs. 1 and 2, respectively. The filter is an acrylic resin cylinder with a diameter of $340 \mathrm{~mm}$, a length of $120 \mathrm{~mm}$ and an effective filtration area of $1180 \mathrm{~cm}^{2}$. The number of filter chambers is 16 and the filtration is carried out in four of them, i. e. $F=0.25$. Filtrate in the filter chamber is discharged through the holes bored in rotary valve (1) and fixed valve (8) in Fig. 2. The filter paper used as the filter medium is ToyoRoshi No. 131 or No. 405 ( $\zeta$-potential is negative). The electrode inside the filter paper and the electrode in the slurry are copper or stainless steel gauze with an opening of $1 \mathrm{~mm}$, and the distance between the two electrodes is $3 \mathrm{~cm}$. The filter chambers are evacuated by hand aspirator (As) and the filtration pressure $\Delta P_{H}$ is kept constant at $10 \mathrm{~cm} \mathrm{Hg}\left(1.33 \times 10^{4} \mathrm{~N} / \mathrm{m}^{2}\right)$ or $20 \mathrm{~cm} \mathrm{Hg}\left(2.67 \times 10^{4} \mathrm{~N} / \mathrm{m}^{2}\right)$ by the regulating valve (Rv). Slurry is fed from the head tank (9) to the slurry tank (7) and the submergence ratio of the drum filter is kept constant by overflowing the slurry. $N$ is 1.04 $( \pm 1.0 \%)$ r.p.m. (the tangential speed is $1.86 \mathrm{~cm} / \mathrm{s}$ ). The filtration time is measured for every constant filtrate volume of $300 \mathrm{~cm}^{3}$ for calcium carbonate slurry, $120 \mathrm{~cm}^{3}$ for white clay slurry and $40 \mathrm{~cm}^{3}$ for bentonite slurry. Temperature changes of both filtrate and slurry with time are measured by the thermistor

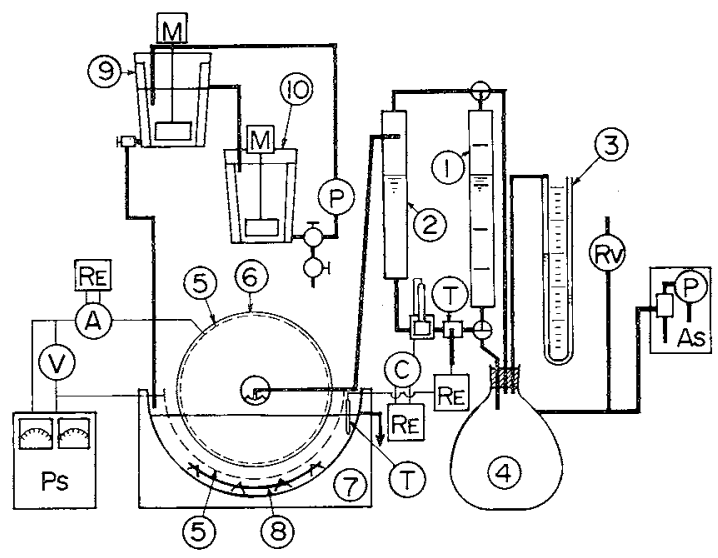
(1) Filtrate measuring cylinder A : Ammeter
(2) Auxiliary measuring cylinder As : Handy aspirator
(3) Mercury manometer $\mathrm{C}$ : Conduct-meter
(4) Filtrate reservoir $\mathrm{M}$ : Motor
(5) Electrode
(6) Filter paper
(7) Slurry tank
(8) Reciprocating agitator
(9) Head tank
(10) Conditioning tank
$\mathrm{P}$ : Pump
Ps : D. C. Power supply
RE : Recorder
RV : Reducing valve
$\mathrm{T}$ : Thermistor thermometer
$\mathrm{V}$ : Voltmeter

Fig. 1 Outline of experimental apparatus for continuous electrokinetic filtration

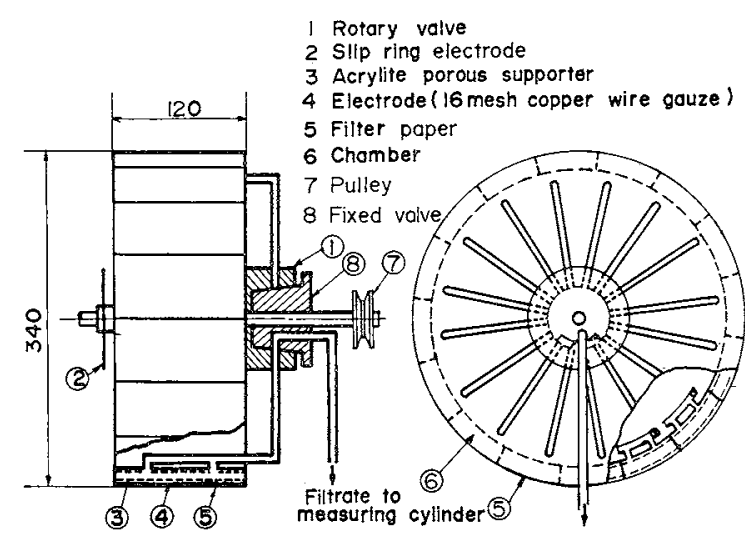

Fig. 2 Detailed diagram of rotary drum filter

thermometer ( $\mathrm{T})$ and the specific conductivity of the filtrate is measured by the conduct meter (C). These values and the value of the electric current are recorded by two recorders $(\mathrm{RE})$. Slurry is gently agitated by the reciprocal agitator so as not to settle. Calcium carbonate (density $\rho_{p}=2.67 \mathrm{~g} / \mathrm{cm}^{3}$, median diameter $d_{p m}=5 \mu \mathrm{m}$ and positive $\zeta$-potential), white clay $\left(\rho_{p}=2.72 \mathrm{~g} / \mathrm{cm}^{3}, d_{p m}=2 \mu \mathrm{m}\right.$ and negative $\zeta$-potential) and bentonite $\left(\rho_{p}=2.48 \mathrm{~g} / \mathrm{cm}^{3}, d_{p m}<1 \mu \mathrm{m}\right.$ and negative $\zeta$-potential) are dispersed in city water as suspended particles. The average concentrations of these slurries $s_{\mathrm{av}}$ are $0.0529( \pm 16 \%)$ for calcium carbonate, $0.0544( \pm 12 \%)$ for white clay and 0.0506 $( \pm 3.0 \%) \mathrm{g}$-solid/g-slurry for bentonite, respectively. The polarity of both electrodes is determined in consideration of the polarity of $\zeta$-potential of particles in 


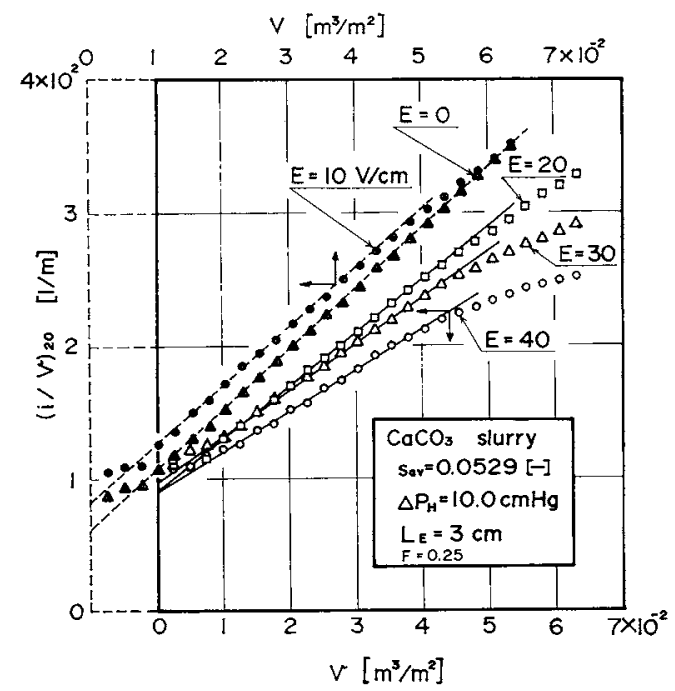

Fig. 3 Relation between $(i / v)_{20}$ and $v$ with strength of electric field as parameter for $\mathrm{CaCO}_{3}$ slurry

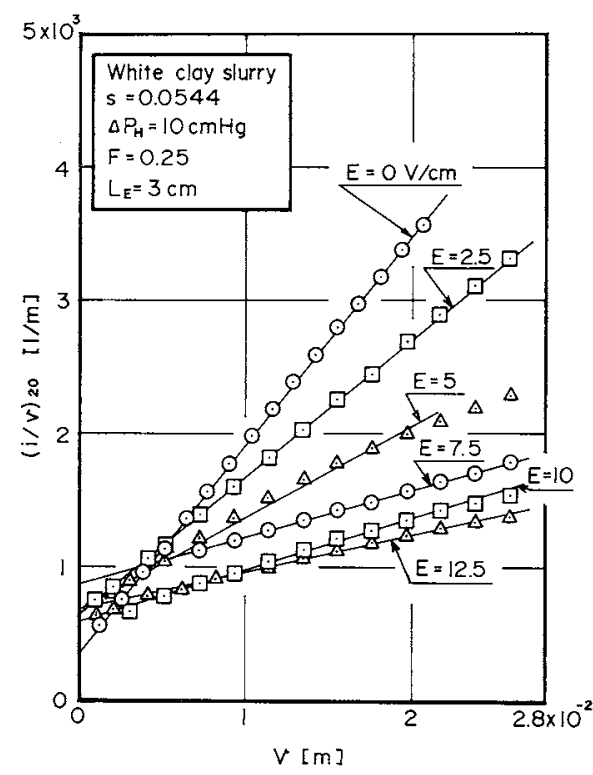

Fig. 4 Relation between $(i / v)_{20}$ and $\boldsymbol{v}$ with strength of electric field as parameter for white clay slurry

order that cake formation may be decreased by electrophoresis in the slurry and the filtrate flow rate may be increased by electroosmosis in the cake. The filter cake is not scraped off during the experiment. After the experiment, sample cake is collected from the cake on the filter medium in order to measure the mass of cake solid per unit filtrate volume $\nu$.

To obtain the values of the average specific filtration resistance $\alpha$ and the electroosmotic filtration pressure $\Delta P_{E}$, another experiment of continuous electrokinetic filtration was performed under the same conditions. The filtration was continued under the filtration pressure $\Delta P_{H}$ alone, after the electric field was cut off in the course of electrokinetic filtration. In the filtration under $\Delta P_{H}, k_{R}$ is got from the experi-

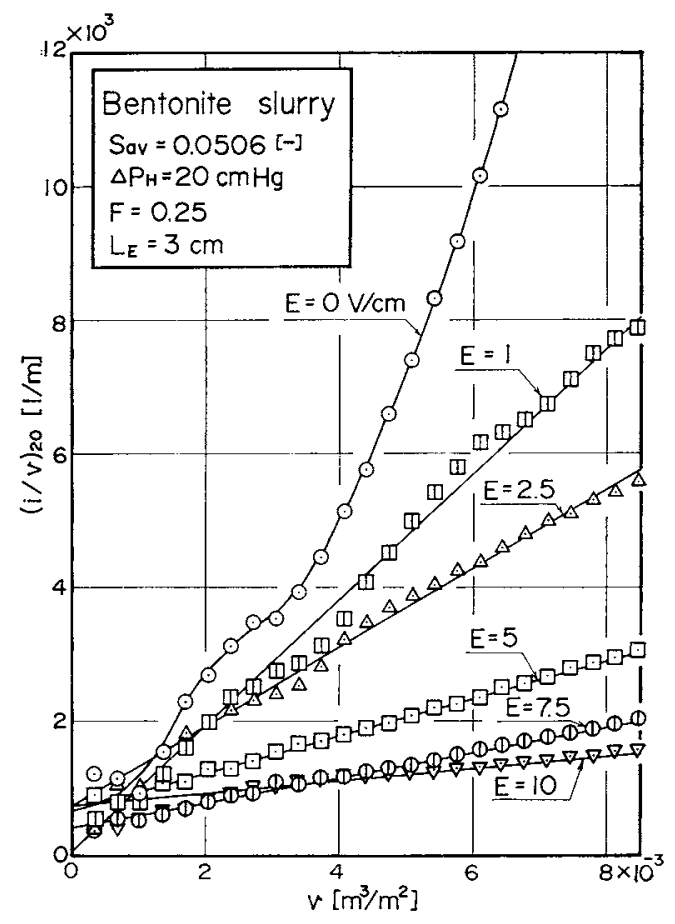

Fig. 5 Relation between $(i / v)_{20}$ and $v$ with strength of electric field as parameter for bentonite slurry

mental relation between $(i / v)_{20}$ and $v$, and $\alpha$ is obtained by the following equation.

$$
\alpha=2 \Delta P_{H} /\left(k_{R} \cdot \mu_{20} \cdot \nu^{*}\right)
$$

By substituting $\alpha, \Delta P_{H}$ and $k_{R}$ obtained from the electrokinetic filtration into the following equation,

$$
\Delta P_{E}=k_{R} \cdot \mu_{20} \cdot \nu^{*} \cdot \alpha / 2-\Delta P_{H}
$$

$\Delta P_{E}$ is estimated assuming that the respective values of $\alpha$ in the former and the latter experiments are equal and constant. In Eqs. (7) and (8), $\nu^{*}$ at a given value of $E$ is calculated by the linear equation fitted to the observed values of $\nu$ and $E$ by the method of least square.

\section{Experimental Results and Discussion}

Figures 3, 4 and 5 show the experimental results of the relation between $(i / v)_{20}$ corrected at $20^{\circ} \mathrm{C}$ and $v$ with the strength of electric field as parameter for calcium carbonate, white clay and bentonite slurries, respectively. The relations between $(i / v)_{20}$ and $v$ are approximately linear for these slurries and each strength of electric field, and it is confirmed that Eq. (5) is practically usable except for the later period of filtration for each slurry and the case at $E=0$ for bentonite slurry, as shown in these figures. The values of $k_{R}$ and $v_{0}$ obtained at $20^{\circ} \mathrm{C}$ for each slurry are listed in Tables 1, 2 and 3 together with the observed values of $\nu$ and other important factors. It is considered that the deviation from the straight line occurred in the later period of filtration because some amount of cake formed on the filter medium was washed off while the 
Table 1 Characteristics of electrokinetic filtration for calcium carbonate slurry

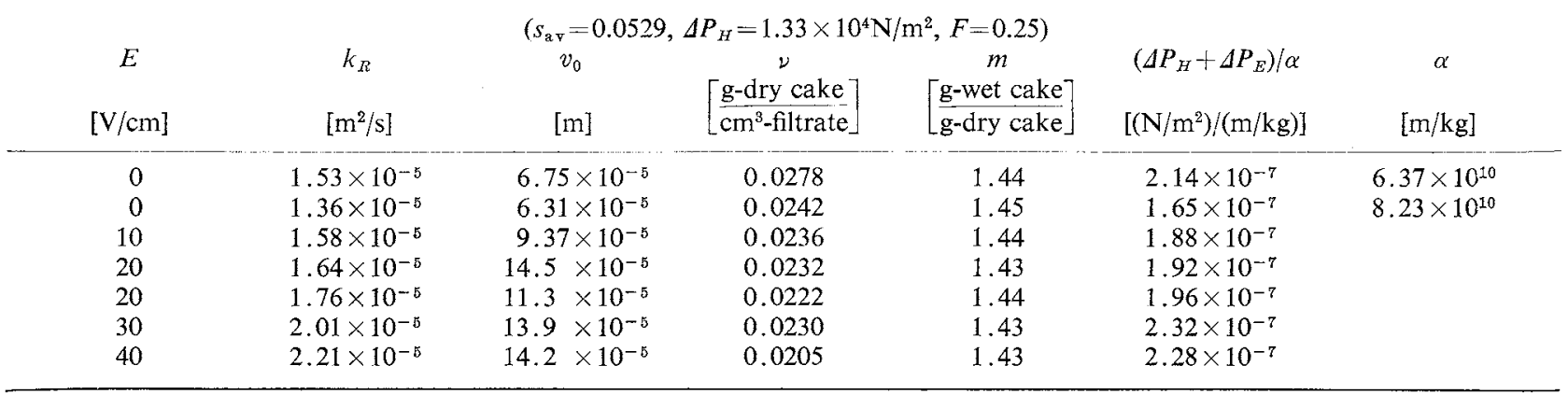

Table 2 Characteristics of electrokinetic filtration for white clay slurry

\begin{tabular}{|c|c|c|c|c|c|c|c|c|}
\hline \multirow{2}{*}{$\begin{array}{c}E \\
{[\mathrm{~V} / \mathrm{cm}]}\end{array}$} & \multirow{2}{*}{$\begin{array}{c}k_{R} \\
{\left[\mathrm{~m}^{2} / \mathrm{s}\right]}\end{array}$} & \multirow[b]{2}{*}{$\begin{array}{c}v_{0} \\
{[\mathrm{~m}]}\end{array}$} & \multicolumn{3}{|c|}{$\left(s_{\mathrm{av}}=0.0544, \Delta P_{H}=1.33 \times 10^{4} \mathrm{~N} / \mathrm{m}^{2}, F=0.25\right)$} & \multirow{2}{*}{$\begin{array}{c}\alpha \\
{[\mathrm{m} / \mathrm{kg}]}\end{array}$} & \multicolumn{2}{|c|}{$\kappa \times 10^{8}[1 / \Omega \cdot \mathrm{cm}]$} \\
\hline & & & {$\left[\frac{\mathrm{g} \text {-dry cake }}{\mathrm{cm}^{3} \text {-filtrate }}\right]$} & {$\left[\begin{array}{c}m \\
\frac{g-w e t ~ c a k e}{\text { g-dry cake }}\end{array}\right]$} & {$\left[\frac{\left(\mathrm{N} / \mathrm{m}^{2}\right)}{(\mathrm{m} / \mathrm{kg})}\right] / \alpha$} & & $\begin{array}{l}\text { values at } \\
V=0 \mathrm{~cm}^{3}\end{array}$ & $\begin{array}{c}1 / \Omega 2 \mathrm{~cm}] \\
\text { values at } \\
V=3000 \mathrm{~cm}^{3}\end{array}$ \\
\hline 0 & $0.425 \times 10^{-8}$ & $.11 \times 10^{-5}$ & 0.0402 & 2.08 & $0.859 \times 10^{-8}$ & $1.55 \times 10^{12}$ & $1.51 \times 10^{2}$ & $2.98 \times 10^{2}$ \\
\hline 2.5 & $0.638 \times 10^{-6}$ & $0.31 \times 10^{-5}$ & 0.0312 & 1.98 & $1.00 \times 10^{-8}$ & & $1.72 \times$ & $6.80 \times 10^{2}$ \\
\hline 5 & $0.960 \times 10^{-6}$ & $0.50 \times 10^{-5}$ & 0.0224 & 2.56 & $1.08 \times 10^{-8}$ & & $1.55 \times 10^{2}$ & $1.27 \times 10^{3}$ \\
\hline 7.5 & $1.88 \times 10^{-8}$ & $1.2 \times 10^{-5}$ & 0.0171 & 2.13 & $1.62 \times 10^{-8}$ & & $1.12 \times 10^{2}$ & $1.63 \times 10^{3}$ \\
\hline 10 & $1.69 \times 10^{-8}$ & $0.75 \times 10^{-5}$ & 0.0103 & 2.14 & $0.952 \times 10^{-8}$ & & $1.90 \times 10^{2}$ & $1.63 \times 10^{3}$ \\
\hline 12.5 & $2.36 \times 10^{-6}$ & $1.2 \times 10^{-5}$ & 0.0101 & 2.11 & $1.20 \times 10^{-8}$ & & $1.47 \times 10^{2}$ & $1.83 \times 10^{3}$ \\
\hline
\end{tabular}

Table 3 Characteristics of electrokinetic filtration for bentonite slurry

\begin{tabular}{|c|c|c|c|c|c|c|}
\hline \multicolumn{7}{|c|}{$\left(s_{\mathfrak{a V}}=0.0506, \Delta P_{H}=2.67 \times 10^{4} \mathrm{~N} / \mathrm{m}^{2}, F=0.25\right)$} \\
\hline$E$ & $k_{R}$ & $v_{0}$ & $\nu$ & $m$ & $\kappa \times 1$ & $\Omega \cdot \mathrm{cm}]$ \\
\hline$[\mathrm{V} / \mathrm{cm}]$ & {$\left[\mathrm{m}^{2} / \mathrm{s}\right]$} & {$[\mathrm{m}]$} & {$\left[\frac{\text { g-dry cake }}{\mathrm{cm}^{3} \text {-filtrate }}\right]$} & {$\left[\frac{\text { g-wet cake }}{\text { g-dry cake }}\right]$} & $\begin{array}{l}\text { values at } \\
V=0 \mathrm{~cm}^{3}\end{array}$ & $\begin{array}{c}\text { values at } \\
V=1000 \mathrm{~cm}^{3}\end{array}$ \\
\hline 0 & & & 0.0276 & 6.66 & $1.33 \times 10^{2}$ & $3.78 \times 10^{2}$ \\
\hline 1 & $0.723 \times 10^{-7}$ & $3.2 \times 10^{-5}$ & 0.0180 & 6.27 & $1.49 \times 10^{2}$ & $5.73 \times 10^{2}$ \\
\hline 2.5 & $1.15 \times 10^{-7}$ & $6.2 \times 10^{-4}$ & & & $1.41 \times 10^{2}$ & $1.45 \times 10^{3}$ \\
\hline 5 & $2.43 \times 10^{-7}$ & $1.2 \times 10^{-3}$ & & & $1.38 \times 10^{2}$ & $1.72 \times 10^{3}$ \\
\hline 7.5 & $3.70 \times 10^{-7}$ & $1.1 \times 10^{-3}$ & & & $1.45 \times 10^{2}$ & $1.80 \times 10^{3}$ \\
\hline 10 & $7.22 \times 10^{-7}$ & $3.9 \times 10^{-3}$ & & & $1.42 \times 10^{2}$ & $2.09 \times 10^{3}$ \\
\hline
\end{tabular}

filter surface was rotating in the slurry, since the structure of cake became loose owing to electric force acting on the particles.

At $E=0$ for bentonite slurry as shown in Fig. 5, Ruth's filtration coefficient $k_{R}$ decreases as cake thickness increases, because the cake formed becomes gelatinous with time and the filtration resistance increases remarkably. It was found that cakes were scarcely deposited on the filter surface with increase of $E$, and the scraping of cake was impossible at more than $E=2.5 \mathrm{~V} / \mathrm{cm}$. At more than $E=2.5 \mathrm{~V} / \mathrm{cm}$, the electric charge of bentonite particles was discharged at the anode and the particles adhered to the slurryside electrode (anode) and the thickness of adherent particles was 0.8 to $1.2 \mathrm{~cm}$, but the slurry concentration almost never changed during the filtration.

According to Tables 1, 2 and 3, for example, the value of $k_{R}$ at $E=40 \mathrm{~V} / \mathrm{cm}$ is 1.6 times as large as that of $k_{R}$ at $E=0 \mathrm{~V} / \mathrm{cm}$ for $\mathrm{CaCO}_{3}$ slurry, $k_{R}$ at $E=12.5 \mathrm{~V} /$ $\mathrm{cm}$ is 5.6 times as large as $k_{R}$ at $E=0$ for white clay slurry, and $k_{R}$ at $E=10 \mathrm{~V} / \mathrm{cm}$ is 10 times as large as $k_{R}$ at $E=1 \mathrm{~V} / \mathrm{cm}$ for bentonite slurry. As described fully in Appendix, the estimated values of the dry cake formation rate per unit filtration area, $d w / d t$, in continuous electrokinetic filtration in which the cake formed is scraped off every revolution are almost the same or are smaller by a maximum of $50 \%$ as compared with that at $E=0$. On the other hand the value of the filtration flow rate $d v / d t$ is a maximum of 2.4 times as large as that at $E=0$ in these experiments. Therefore, it is found that the effect of the electric field on filtration flow rate is remarkable and that electrokinetic filtration is usable for slurries suspended with fine particles and for the slurry forming gelatinous cake. It was observed that the suspended particles of calcium carbonate were more coagulated with increase of $E$.

Figure 6 shows the relation between $\left(\Delta P_{H}+\Delta P_{E}\right)$ / $\alpha$ and $E$ and the relation between values of $\nu$ and $E$ for $\mathrm{CaCO}_{3}$ slurry, which are listed in Table 1. Figure 7 shows the relation between values of $\nu$ and $E$ for white clay slurry, which are listed in Table 2 . According to Figs. 6 and 7, relations between $\nu$ and $E$ for both $\mathrm{CaCO}_{3}$ and white clay slurries are linear and can be represented by the equation $\nu=\nu_{0}\left(1-E / E_{c r}\right)^{7}$. The linear equations obtained by the method of least 


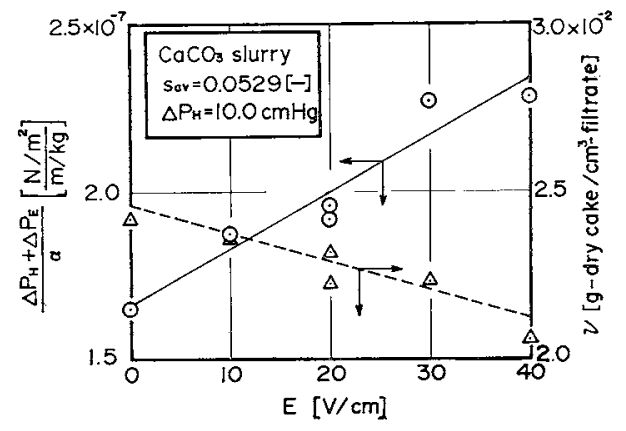

Fig. 6 Relations between $\left(\Delta P_{H}+\Delta P_{E}\right) / \alpha$ and $E$ and between $\nu$ and $E$ for $\mathrm{CaCO}_{3}$ slurry

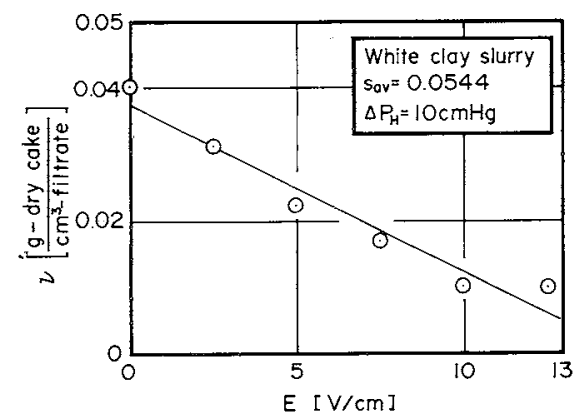

Fig. 7 Relation between $\nu$ and $E$ for white clay slurry

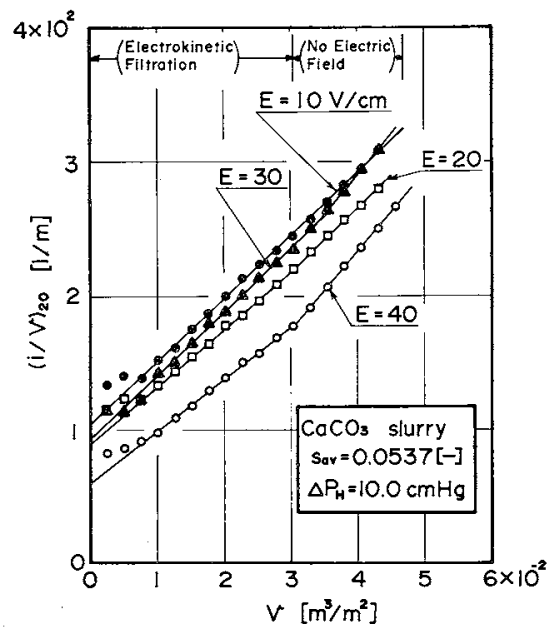

Fig. \& Experimental results of electrokinetic filtration and successive constant hydraulic pressure filtration for $\mathrm{CaCO}_{3}$ slurry

square, shown as the lines in Figs. 6 and 7, are expressed by the following experimental formula:

$$
\begin{gathered}
\nu=0.0244(1-E / 305), E_{c r}=305 \mathrm{~V} / \mathrm{cm} \\
\text { for } \mathrm{CaCO}_{3} \text { slurry } \\
\nu=0.0375(1-E / 15.0), E_{c r}=15.0 \mathrm{~V} / \mathrm{cm}
\end{gathered}
$$

for white clay slurry

Figure 8 shows the relation between $(i / v)_{20}$ and $v$ for the electrokinetic filtration and the successive constant hydraulic pressure filtration in order to evaluate the values of the electrokinetic filtration pressure $\Delta P_{E}$ and

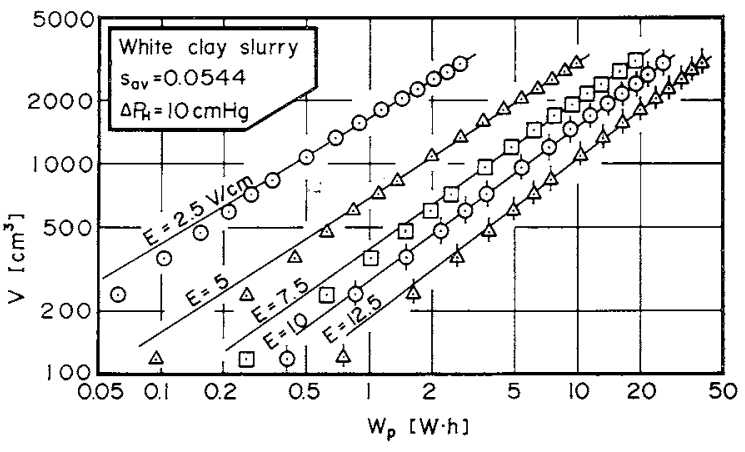

Fig. 9 Relations between $V$ and $W_{p}$ with strength of electric field as parameter for white clay slurry

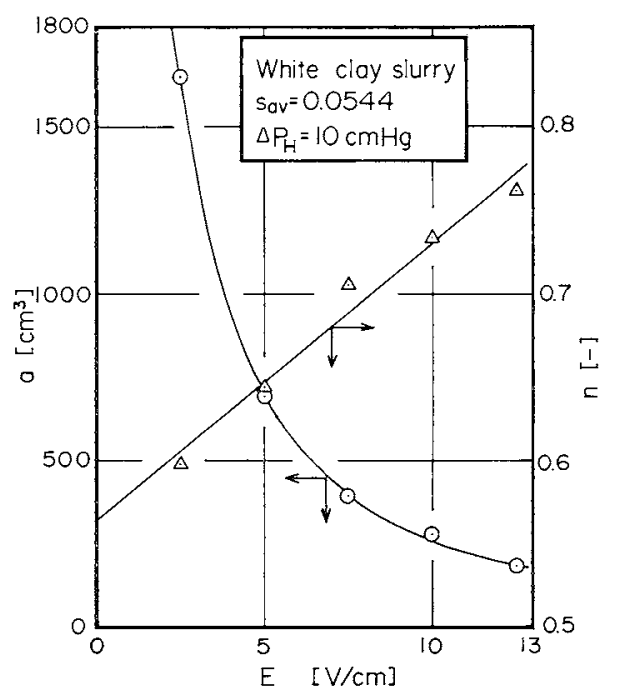

Fig. 10 Changes in $a$ and $n$ in empirical equation of $V=a \cdot W_{p}{ }^{n}$ owing to $E$ for white clay slurry

the average specific filtration resistance $\alpha$ in the electrokinetic filtration for the slurry of $\mathrm{CaCO}_{3}$. The obtained characteristics of electrokinetic filtration are listed in Table 4. In the relation between $\alpha$ and $E$ for batch electrokinetic filtration, $\alpha$ decreases as $E$ increases $^{7}$, but in this experiment the correlation between $\alpha$ and $E$ is not clear. In the relation between $\Delta P_{E}$ and $E$ for batch electrokinetic filtration, $\Delta P_{E}$ is proportional to $E^{7}$. In this experiment $\Delta P_{E}$ increases as $E$ increases, but $\Delta P_{E}$ is not directly proportional to $E$.

Figure 9 shows the relation between $V$ and the electric power consumption $W_{p}$ with $E$ as parameter for white clay slurry. According to Fig. 9, the relationship between $V$ and $W_{p}$ for each value of $E$ can be represented by the empirical equation $V=a \cdot W_{p}{ }^{n}$, similarly to batch electrokinetic filtration ${ }^{7)}$, in which $a$ and $n$ are experimental constants. Similar empirical equations are obtained for $\mathrm{CaCO}_{3}$ slurry and bentonite slurry. Table 5 shows the observed values of $a$ and $n$ for each slurry and for each value of $E$. Figure $\mathbf{1 0}$ shows, as an example, plots of $a$ and $n$ against $E$ for 
Table 4 Characteristics of electrokinetic filtration for calcium carbonate slurry

\begin{tabular}{|c|c|c|c|c|c|c|c|c|}
\hline $\begin{array}{c}E \\
{[\mathrm{~V} / \mathrm{cm}]}\end{array}$ & $\begin{array}{c}k_{R} \\
{\left[\mathrm{~m}^{2} / \mathrm{s}\right]}\end{array}$ & $\begin{array}{l}v_{0} \\
{[\mathrm{~m}]}\end{array}$ & $\begin{array}{c}\left(s_{\mathrm{av}}=0.053\right. \\
k_{R 0} \\
{\left[\mathrm{~m}^{2} / \mathrm{s}\right]}\end{array}$ & $\begin{array}{l}, \Delta P_{H}=1.33 \times \\
\nu^{*} \\
{\left[\frac{\mathrm{g} \text {-dry cake }}{\mathrm{cm}^{3} \text {-filtrate }}\right]}\end{array}$ & $\begin{array}{c}10^{4} \mathrm{~N} / \mathrm{m}^{2}, F=0.2 \\
\left(\Delta P_{H}+\Delta P_{E}\right) / \alpha \\
{\left[\frac{\left(\mathrm{N} / \mathrm{m}^{2}\right)}{(\mathrm{m} / \mathrm{kg})}\right]}\end{array}$ & $\begin{array}{c}\Delta P_{H} / \alpha \\
{\left[\frac{\left(\mathrm{N} / \mathrm{m}^{2}\right)}{(\mathrm{m} / \mathrm{kg})}\right]}\end{array}$ & $\begin{array}{c}\alpha \\
{[\mathrm{m} / \mathrm{kg}]}\end{array}$ & $\begin{array}{c}\Delta P_{E} \\
{\left[\mathrm{~N} / \mathrm{m}^{2}\right]}\end{array}$ \\
\hline 0 & $1.36 \times 10^{-5}$ & $6.31 \times 10^{-5}$ & $1.36 \times 10^{-5}$ & 0.0244 & $1.65 \times 10^{-7}$ & $1.65 \times 10^{-7}$ & $8.23 \times 10^{10}$ & 0 \\
\hline 10 & $1.49 \times 10^{-5}$ & $11.2 \times 10^{-5}$ & $1.39 \times 10^{-5}$ & 0.0236 & $1.77 \times 10^{-7}$ & $1.70 \times 10^{-7}$ & $7.84 \times 10^{10}$ & $5.5 \times 10^{2}$ \\
\hline 20 & $1.60 \times 10^{-5}$ & $10.6 \times 10^{-5}$ & $1.42 \times 10^{-5}$ & 0.0228 & $1.83 \times 10^{-7}$ & $1.74 \times 10^{-7}$ & $7.66 \times 10^{10}$ & $7.0 \times 10^{2}$ \\
\hline 30 & $1.46 \times 10^{-5}$ & $9.84 \times 10^{-5}$ & $1.17 \times 10^{-5}$ & 0.0220 & $1.61 \times 10^{-7}$ & $1.44 \times 10^{-7}$ & $9.28 \times 10^{10}$ & $16.3 \times 10^{2}$ \\
\hline 40 & $1.76 \times 10^{-5}$ & $7.70 \times 10^{-5}$ & $1.17 \times 10^{-5}$ & 0.0212 & $1.88 \times 10^{-7}$ & $1.43 \times 10^{-7}$ & $9.29 \times 10^{10}$ & $41.2 \times 10^{2}$ \\
\hline
\end{tabular}

Table 5 Values of $\boldsymbol{a}$ and $\boldsymbol{n}$ in $\boldsymbol{V}=\boldsymbol{a} \cdot \boldsymbol{W}_{p}{ }^{n}$

\begin{tabular}{|c|c|c|c|c|c|c|c|c|}
\hline \multicolumn{6}{|c|}{$\begin{array}{c}\text { ( } V \text { expressed in } \mathrm{cm}^{3} \text { and } W_{p} \text { in watt-hr) } \\
\text { White clay }\left(s_{a,}=0.0544\right)\end{array}$} & \multicolumn{3}{|c|}{ Bentonite $\left(s_{\mathrm{av}}=0.0506\right)$} \\
\hline $\begin{array}{c}E \\
{[\mathrm{~V} / \mathrm{cm}]}\end{array}$ & $\begin{array}{c}a \\
{\left[\mathrm{~cm}^{3}\right]}\end{array}$ & {$[-n$} & $\begin{array}{c}E \\
{[\mathrm{~V} / \mathrm{cm}]}\end{array}$ & $\begin{array}{c}a \\
{\left[\mathrm{~cm}^{3}\right]}\end{array}$ & {$[-]$} & $\begin{array}{c}E \\
{[\mathrm{~V} / \mathrm{cm}]}\end{array}$ & {$\left[\begin{array}{c}a \\
{\left[\mathrm{~cm}^{3}\right]}\end{array}\right.$} & {$[-]$} \\
\hline 10 & 1710 & 0.675 & 2.5 & 1650 & 0.598 & 1 & 1270 & 0.512 \\
\hline 20 & 636 & 0.734 & 5 & 689 & 0.644 & 2.5 & 397 & 0.563 \\
\hline 20 & 513 & 0.767 & 7.5 & 393 & 0.706 & 5 & 222 & 0.606 \\
\hline 30 & 310 & 0.814 & 10 & 278 & 0.734 & 7.5 & 162 & 0.610 \\
\hline 40 & 153 & 0.873 & 12.5 & 183 & 0.762 & 10 & 89.7 & 0.697 \\
\hline
\end{tabular}

white clay slurry. According to Fig. $10, n$ increases linearly and $a$ decreases like a hyperbolic curve as $E$ increases. The correlation between $n$ and $E$ and the correlation between $a$ and $E$ for other slurries are similar to those for white clay slurry.

The specific electric conductivity $\kappa$ of filtrate of $\mathrm{CaCO}_{3}$ slurry increased by $20 \%$ compared with that of city water used in this experiment, but $\kappa$ of filtrate of white clay and bentonite slurries increased by $2-15$ times that of city water with increase of $E$ as shown in Tables 2 and 3 . These results correspond with the fact that electrokinetic filtration of slurry having higher specific electric conductivity consumes more electric power. For example, the values of $W_{p}$ required to obtain a filtrate volume of $1000 \mathrm{~cm}^{3}$ at $E=10 \mathrm{~V} / \mathrm{cm}$ were 0.46 watt-hour for $\mathrm{CaCO}_{3}, 5.8$ for white clay and 32 for bentonite, respectively.

\section{Conclusion}

In this study on continuous electrokinetic filtration with a modified rotary drum vacuum filter of the Oliver type, the following results have been confirmed.

1) The fundamental equation for electrokinetic filtration with the rotary vacuum filter, taking account of electrophoresis and electroosmosis, is represented by Eq. (3) and is of the same form as Ruth's equation for constant-pressure filtration. But Ruth's coefficient $k_{R}$ of electrokinetic filtration is not only a function of $\Delta P_{H}$ but also a function of the electric field. The equation for electrokinetic filtration using a rotary drum vacuum filter is given by Eq. (5).

2) Electrokinetic filtration is usable to increase the filtration flow rate for slurries such as bentonite, which is difficult to filter mechanically because of gelatinous cake formation and an electrokinetic filter made by modifying a filter of the Oliver type is one type of practical equipment.

3) The relation between filtrate volume $V$ and electric power consumption $W_{p}$ in continuous electrokinetic filtration is represented by the empirical equation $V=a \cdot W_{p}^{n}$, similar to that of batch electrokinetic filtration, in which $a$ and $n$ are experimental constants depending on the kind of slurry and are functions of $E$.

Appendix: Estimated values of $d v / d t$ and $d w / d t$ in continuous electrokinetic filtration

The filtration flow rate $d v / d t$ and the dry cake formation rate $d w / d t$ per unit filtration area in continuous electrokinetic filtration in which the cake formed is scraped off every revolution are shown in Table A-1. These values in Table A-1 were calculated by Eq. (6) in the case of $i=1, F=0.25$ and $N=1.04 \mathrm{rpm}$. According to Table A-1, in the case of calcium carbonate slurry, the values of $d w / d t$ are almost the same or are smaller by $14 \%$ (maximum) as compared with that at $E=0$. On the other hand the value of $d v / d t$ at $E=40 \mathrm{~V} / \mathrm{cm}$ is larger than that at $E=0$ by $27 \%$ (maximum). In the case of white clay slurry, the values of $d w / d t$ in the range of $E=2.5-7.5 \mathrm{~V} / \mathrm{cm}$ are almost the same as that at $E=0$, or $d w / d t$ at $E=10 \mathrm{~V} / \mathrm{cm}$ is smaller than that at $E=0$ by $50 \%$ (maximum), but the value of $d v / d t$ at $E=12.5 \mathrm{~V} / \mathrm{cm}$ is 2.4 times that at $E=0$. For bentonite slurry, the values of $d w / d t$ cannot be compared because of small cake formation, but the value of $d v / d t$ at $E=10 \mathrm{~V} / \mathrm{cm}$ is about twice that at $E=0$.

$d w / d t=\nu(d v / d t)$ is calculated by Eq. (A-1), substituting Eq. (4) into Eq. (6) and neglecting the filter medium resistance.

$$
\frac{d w}{d t}=\sqrt{\frac{\left(\Delta P_{H}+\Delta P_{E}\right) \nu}{2 \mu \alpha} \cdot N}
$$

According to the authors' investigation ${ }^{7)}$, Tables 1 and 4 and Fig. $6, \Delta P_{E}$ increases nearly in proportion to $E$, $\nu$ decreases linearly and $\alpha$ decreases hyperbolically with increasing $E$. Therefore, it does not necessarily follow that the value of $d w /$ $d t$ diminishes theoretically by applying an electric field to constant-pressure filtration.

\section{Acknowledgment}

This work was supported by a Grant-in-Aid for Scientific Research from the Ministry of Education, Japan. The authors 
Table A-1 Values of $d v / d t$ and $d w / d t$ calculated by Eq. (6) as $i=1, F=0.25$ and $N=1.04$ r.p.m.

1) Calcium carbonate slurry

\begin{tabular}{|c|c|c|c|c|c|c|}
\hline & $\begin{array}{c}E \\
{[\mathrm{~V} / \mathrm{cm}]}\end{array}$ & $\begin{array}{c}k_{R} \\
{\left[\mathrm{~m}^{2} / \mathrm{s}\right]}\end{array}$ & $\begin{array}{c}v_{0} \\
{[\mathrm{~m}]}\end{array}$ & {$\left[\frac{\mathrm{g}-\mathrm{dry} \text { cake }^{2}}{\mathrm{~cm}^{3} \text {-filtrate }}\right]$} & $\begin{array}{c}d v / d t \\
{\left[\mathrm{~m}^{3} / \mathrm{m}^{2} \cdot \mathrm{hr}\right]}\end{array}$ & $\begin{array}{c}d w / d t \\
{\left[\mathrm{~kg} / \mathrm{m}^{2} \cdot \mathrm{hr}\right]}\end{array}$ \\
\hline & 0 & $1.53 \times 10^{-5}$ & $6.75 \times 10^{-5}$ & 0.0278 & 1.85 & 51.5 \\
\hline & 0 & $1.36 \times 10^{-5}$ & $6.31 \times 10^{-5}$ & 0.0242 & 1.75 & 42.3 \\
\hline & 10 & $1.58 \times 10^{-5}$ & $9.37 \times 10^{-5}$ & 0.0236 & 1.88 & 44.5 \\
\hline & 20 & $1.64 \times 10^{-5}$ & $14.5 \times 10^{-5}$ & 0.0232 & 1.92 & 44.5 \\
\hline & 20 & $1.76 \times 10^{-5}$ & $11.3 \times 10^{-5}$ & 0.0222 & 1.99 & 44.1 \\
\hline & 30 & $2.01 \times 10^{-5}$ & $13.9 \times 10^{-5}$ & 0.0230 & 2.12 & 48.9 \\
\hline & 40 & $2.21 \times 10^{-5}$ & $14.2 \times 10^{-5}$ & 0.0205 & 2.23 & 45.7 \\
\hline \multirow[t]{8}{*}{ 2) } & $\begin{array}{l}\text { White clay slurry } \\
E\end{array}$ & $k_{R}$ & $v_{0}$ & $\lceil$ g-dry cake $\rceil$ & $d v / d t$ & $d w / d t$ \\
\hline & {$[\mathrm{V} / \mathrm{cm}]$} & {$\left[\mathrm{m}^{2} / \mathrm{s}\right]$} & [m] & {$\left[\mathrm{cm}^{3}\right.$-filtrate } & {$\left[\mathrm{m}^{3} / \mathrm{m}^{2} \cdot \mathrm{hr}\right]$} & {$\left[\mathrm{kg} / \mathrm{m}^{2} \cdot \mathrm{hr}\right]$} \\
\hline & 0 & $0.425 \times 10^{-6}$ & $0.11 \times 10^{-5}$ & 0.0402 & 0.309 & 12.4 \\
\hline & 2.5 & $0.638 \times 10^{-6}$ & $0.31 \times 10^{-5}$ & 0.0312 & 0.378 & 11.8 \\
\hline & 5 & $0.960 \times 10^{-8}$ & $0.50 \times 10^{-5}$ & 0.0224 & 0.464 & 10.4 \\
\hline & 7.5 & $1.88 \times 10^{-6}$ & $1.2 \times 10^{-5}$ & 0.0171 & 0.650 & 11.1 \\
\hline & 10 & $1.69 \times 10^{-8}$ & $0.75 \times 10^{-5}$ & 0.0103 & 0.616 & 6.35 \\
\hline & 12.5 & $2.36 \times 10^{-6}$ & $1.2 \times 10^{-5}$ & 0.0101 & 0.728 & 7.35 \\
\hline \multirow[t]{4}{*}{ 3) } & Bentonite slurry & & & & & \\
\hline & $E$ & $k_{R}$ & $v_{0}$ & $\stackrel{\nu}{[\text { g-dry cake }]}$ & $d v / d t$ & $d w / d t$ \\
\hline & {$[\mathrm{V} / \mathrm{cm}]$} & {$\left[\mathrm{m}^{2} / \mathrm{s}\right]$} & {$[\mathrm{m}]$} & {$\left[\mathrm{cm}^{3}\right.$-filtrate $]$} & {$\left[\mathrm{m}^{3} / \mathrm{m}^{2} \cdot \mathrm{hr}\right]$} & {$\left[\mathrm{kg} / \mathrm{m}_{\lambda}^{2} \cdot \mathrm{hr}\right]$} \\
\hline & 0 & & & 0.0276 & & \\
\hline \multirow[t]{5}{*}{$=$} & 1 & $0.723 \times 10^{-7}$ & $3.2 \times 10^{-5}$ & 0.0180 & 0.127 & 2.29 \\
\hline & 2.5 & $1.15 \times 10^{-7}$ & $6.2 \times 10^{-4}$ & & 0.145 & \\
\hline & 5 & $2.43 \times 10^{-7}$ & $1.2 \times 10^{-3}$ & & 0.197 & \\
\hline & 7.5 & $3.70 \times 10^{-7}$ & $1.1 \times 10^{-3}$ & & 0.260 & \\
\hline & 10 & $7.22 \times 10^{-7}$ & $3.9 \times 10^{-3}$ & & 0.257 & \\
\hline
\end{tabular}

are grateful to Messrs. Yutaka Tsukui, Itsunaga Miyahara and Kan-ichi Yamaguchi for their assistance in this work.

Nomenclature

A $\quad=$ filtration area

$a \quad=$ experimental constant

$d_{p m} \quad=$ median diameter of particles in slurry

$E \quad=$ strength of electric field

$E_{c r} \quad=$ critical strength of electric field

$F \quad=$ subnergence ratio of drum filter

$i \quad=$ number of revolution

$K_{R} \quad=$ modified Ruth's filtration coefficient

$k_{R} \quad=$ modified Ruth's filtration coefficient divided by square of filtration area

$k_{R 0} \quad=$ value of $k_{R}$ after cutting off electric field in experiment made for evaluation of $\alpha$ and $\Delta P_{E}$

$L_{E^{\prime}} \quad=$ distance between electrodes

$m \quad=$ ratio of mass of wet to dry cake

$N \quad=$ revolutions per unit time

$n \quad=$ experimental constant

$\Delta P_{E} \quad=$ electroosmotic filtration pressure

$\Delta P \quad=$ hydraulic filtration pressure $[\mathrm{cm} \mathrm{Hg}]$ or $\left[\mathrm{N} / \mathrm{m}^{2}\right]$

$R_{m} \quad=$ filter medium resistance $\quad[1 / \mathrm{m}]$

$s_{\mathrm{av}}=$ average slurry concentration [g-solid/g-slurry]

$t \quad=$ filtration time

$t_{r} \quad=$ real filtration time in continuous filtration

$V \quad=$ filtrate volume

$V_{0} \quad=$ fictitious filtrate volume

$v \quad=$ filtrate volume per unit filtration area

$v_{0} \quad=$ fictitious filtrate volume per unit filtration

$[\mathrm{V} / \mathrm{cm}]$
$[\mathrm{V} / \mathrm{cm}]$

$[-]$

$\left[\mathrm{m}^{6} / \mathrm{s}\right]$

$\left[\mathrm{m}^{2} / \mathrm{s}\right]$

$\left[\mathrm{m}^{2} / \mathrm{s}\right]$

$1 / \mathrm{s}]$ or $[1 / \mathrm{min}]$

$[-]$

$\left.\mathrm{N} / \mathrm{m}^{2}\right]$

[s]

$\left[\mathrm{m}^{\hat{3}}\right]$

$\left[\mathrm{m}^{3}\right]$

[m] area

$W_{\mathrm{p}} \quad=$ electric power consumption

[m]

$w$

$=$ dry cake mass per unit filtration area

[watt-hr]

$\left[\mathrm{kg} / \mathrm{m}^{2}\right]$

$=$ average specific filtration resistance

$[\mathrm{m} / \mathrm{kg}]$

$=\zeta$-potential of particles

$=$ specific electric conductivity

$=$ viscosity of filtrate

= dry cake mass per unit volume of filtrate in electrokinetic filtration

$[\mathrm{kg} / \mathrm{m} \cdot \mathrm{s}]$

$[-]$

[cm]

$[-]$

[s]

[g-solid $/ \mathrm{cm}^{3}$-filtrate]

$=\nu$ in the absence of electric field

[g-solid $/ \mathrm{cm}^{3}$-filtrate]

$=$ value of $\nu$ fitted by method of least square

[g-solid/ $/ \mathrm{cm}^{3}$-filtrate]

$\rho_{p}$

$=$ density of solid particles

$\left[\mathrm{g} / \mathrm{cm}^{3}\right]$

\section{Literature Cited}

1) Fujita, S.: "Kagaku Kōgaku Enshū”,, p. 230, TokyoKagaku-Dōjin, Tokyo (1967).

2) Henry, J. D., L. F. Lawler and C. H. A. Kuo: AIChE J., 23, 851 (1977).

3) Moulik, S. P., F. C. Cooper and M. Bier: J. Colloid Interface Sci., 24, 427 (1967).

4) Moulik, S. P.: Environ. Sci. Technol., 5, 771 (1971).

5) Shirato, M., T. Murase and K. Kobayashi: Filtration Separation, 5, 219 (1968).

6) Yoshida, F. and Y. Mori: "Shōron Kagaku Kögaku I", p. 399, Asakura-Shoten, Tokyo (1968).

7) Yukawa, H., K. Kobayashi, Y. Tsukui, S. Yamano and M. Iwata: J. Chem. Eng. Japan, 9, 396 (1976). 